\title{
Comparison of Various Window Functions Used in FIR Filter Designing \\ Shruti Jain ${ }^{1}$ \\ P.D.M. College of Engineering, Bahadurgarh, Haryana (India)
}

shrutier308@gmail.com ${ }^{1}$

Mr. Dinesh Kumar Verma²

P.D.M. College of Engineering, Bahadurgarh, Haryana (India)

erdineshverma@gmail.com ${ }^{2}$

\section{ABSTRACT}

In this study, 2-parameter cosh window is modified to improve its spectral characteristic in terms of the ripple ratio by proposing a new additional parameter. It is observed that an increase in the new parameter results in wider mainlobe width and smaller ripple ratio. By choosing the suitable combinations of the two adjustable parameters for the proposed modified window, the optimum windows that yield the minimum ripple ratio for $\mathrm{N}=51$ and $\mathrm{N}=101$ are .found. Simulation results show that the filters designed by proposed 3-parameter Cosh window provide higher quality in terms of the minimum stop-band attenuation for a fixed order, and yield lower order for a fix minimum stop-band attenuation compared to the filters designed by other windows.

Keywords: Modified Cosh window; exponential window; Kaiser window; ripple-ratio; FIR filter design; side-lobe roll-off ratio; ripple ratio;

\section{Council for Innovative Research}

Peer Review Research Publishing System

Journal: INTERNATIONAL JOURNAL OF COMPUTERS \& TECHNOLOGY

Vol 8, No 3 


\section{INTRODUCTION}

Digital filters are the most important and frequently used elements in digital signal processing applications. These are classified as finite impulse response (FIR) and infinite impulse response (IIR) filters based on the duration of their impulse response. Both of these filters have advantages and disadvantages. Therefore, neither of them can be considered as best for all situations. The advantages of FIR filter are that these are most popular, always stable and these have linear phase but it has a disadvantage of FIR filters over IIR filters which is their implementation complexity in case the filter order is very large. The implementation of FIR filters with nonrecursive techniques guarantees stability [1].

A more comprehensive view of the truncation and smoothing operations is in terms of window functions (or windows for short). Windows are frequently compared and classified in terms of their spectral characteristics. Window functions are widely used for different applications of Digital Signal processing such as signal analysis, signal estimation, Digital filter design and speech processing [1]-[2]. Number of windows has been proposed for different applications [3]-[6]. They are having the suboptimal solutions and depending upon the applications. Due to their flexibility properties, the adjustable windows such as Kaiser and Dolph-Chebyshev windows are very attractive in signal processing applications. Cosh window [6], is a two-parameter window, and achieves better sidelobe characteristic than Kaiser and Dolph-Chebyshev windows by adjusting its two independent parameters, namely the window length and the adjustable shape parameter.

The most popular windows for designing filters are ultraspherical [3], Kaiser [5], Saramaki [9], and DolphChebyshev [8], recently cosh [10], Exponential [11] and Modified Kaiser window [12]. The adjustable windows except the ultraspherical window have two parameters.

It is investigated in [3] that the ultraspherical window family provides the highest minimum stop-band attenuation for a given fixed filter length and yields the lowest filter length to satisfy the given stop-band attenuation as compared to the windows in literature.

Note that if $\mathrm{N}$ denotes the filter length, then the filter order is defined as $\mathrm{N}-\mathrm{I}$.

The far-end stop-band attenuation is very important parameter when the filter is designed for the speech processing and sub-band coding and this parameter should be more so that energy leak from one band to another band could be minimum [7]. FIR filter designed by Kaiser window has a better far-end stop-band attenuation than filter designed by the other previously well known adjustable windows such as Dolph-Chebyshev and Saramaki, which are special cases of Ultraspherical window, but obtaining a digital filter which performs higher far-end stop-band attenuation than Kaiser window will be useful. So in this paper nonrecursive digital FIR filter design by 3-parameter Cosh window is proposed which provides better far-end stop-band attenuation than filter designed by well known Kaiser window which is the figure of merit.

2-parameter Cosh window is modified by introducing third parameter so as to provide a more flexible and useful window family for designing higher quality nonrecursive digital filters in terms of the minimum

stopband attenuation, low filter order and more far-end stopband attenuation.

\section{DIFFERENT WINDOWS}

\subsection{KAISER WINDOW}

The window function for Kaiser window is expressed as

$$
\mathrm{w}_{\mathrm{mk}}(\mathrm{n})=\left\{\left[\frac{\mathrm{I}_{0}\left(\mathrm{a}_{\mathrm{mk}} \sqrt{1-\left(\frac{2 \mathrm{n}}{\mathrm{N}-1}\right)^{2}}\right)}{\mathrm{I}_{0}\left(\mathrm{a}_{\mathrm{mk}}\right)} \quad|\mathrm{n}| \leq \frac{(\mathrm{N}-1)}{2}\right]\right.
$$

\subsection{COSH WINDOW}

Cosh window is defined by [6]:

$$
w[n]=\frac{\cosh \left(\alpha \sqrt{1-\left(\frac{2 n}{N-1}\right)^{2}}\right)}{\cosh (\alpha)} ; 0 \leq n \leq(N-1)
$$

where $\alpha c$ and $\mathrm{N}$ are the adjustable parameters to control the window spectrum in terms of the ripple ratio and mainlobe width. In [6], it is shown that for a fixed window length and mainlobe width, cosh window performs better sidelobe roll-off ratio but worse ripple ratio compared to Kaiser window. 


\subsection{MODIFIED COSH WINDOW}

By introducing the third parameter, $\rho$ mc in 2-parameter Cosh window, the 3-parameter Cosh window can be obtained which is defined as[13-14]

$$
w[n]=\left(\frac{\cosh \left(\alpha \sqrt{1-\left(\frac{2 n}{N-1}\right)^{2}}\right)}{\cosh (\alpha)}\right)^{\rho} ; 0 \leq n \leq(N-1)
$$

Note that $\rho \mathrm{mc}=0$ and $\rho \mathrm{mc}=1$ correspond to the Rectangular and 2-parameter Cosh window, respectively.

When the shape characteristic of $\left[\cosh (X)^{\mathrm{pmc}}\right]$ in 3- parameter Cosh window) is compared with $\cosh (\mathrm{x})$ (used in 2 parameter Cosh window) and $\mathrm{lo}(\mathrm{x})$ (used in Kaiser window), then it is found that the shape characteristics of function $\cosh (\mathrm{x})^{\mathrm{pmc}}, \cosh (\mathrm{x}), \exp (\mathrm{x})$ and $\mathrm{lo}(\mathrm{x})$ are same. The simulation of shape characteristics of these functions are shown in Fig. 1 Therefore, 3-parameter Exponential window can be proposed as (I).

Having three parameters makes the modified cosh window more flexible compared to 2-parameter cosh window.

\subsection{EXPONENTIAL WINDOW}

$$
w[n]=\frac{\exp \left(\alpha \sqrt{1-\left(\frac{2 n}{N-1}\right)^{2}}\right)}{\exp (\alpha)} ; 0 \leq n \leq(N-1)
$$

\subsection{ULTRASPHERICAL WINDOW}

The coefficients of the ultraspherical window can be calculated as [5].

$$
\omega(n T)=\frac{\mu x_{\mu}^{2 M}}{M+|n|}\left(\begin{array}{c}
\mu+M+|n|-1 \\
M+|n|-1
\end{array}\right)
$$

$$
\begin{aligned}
& \sum_{m=0}^{M-|n|}\left(\begin{array}{c}
\mu+M-|n|-1 \\
M-|n|-m
\end{array}\right) \\
& \cdot\left(\begin{array}{c}
M+|n| \\
m
\end{array}\right) A^{m} \text { for } n \leq M
\end{aligned}
$$

\section{WINDOW METHOD FOR FIR FILTER DESIGNED BY PROPOSED WINDOW}

A window $\mathrm{w}(\mathrm{nT})$ with a length of $\mathrm{N}$ is nonzero for $|n| \leq \frac{N-1}{2}$ and zero for otherwise [2]. The impulse

response of a realizable non casual filter using a window is obtained as[14]:

$$
h_{n c}(n T)=w(n T) h_{i d}(n T)
$$

Where $h_{i d}(n T)$ is the infinite duration impulse response of the ideal filter. For a low pass filter with a cut off frequency, $\omega$ and sampling frequency, ws, it can be found as [2].

$$
h_{i d}(n T)=\left\{\begin{array}{c}
\frac{\omega_{c} T}{\pi} ; n=0 \\
\frac{\sin \omega_{c} n T}{n \pi} ; \omega_{c} \leq|\omega| \leq \omega_{s} / 2
\end{array}\right.
$$




$$
h(n T)=h_{n c}\left[\left(n-\frac{N-i}{2}\right) T\right] ; 0 \leq n \leq N-1
$$

The transition width (t.w) and cut-off frequency for the given passband $\left(\omega_{p}\right)$ and stopband $\left(\omega_{s t}\right)$ frequencies, are defmed by

$$
\Delta \omega=\omega_{s t}-\omega_{p}
$$

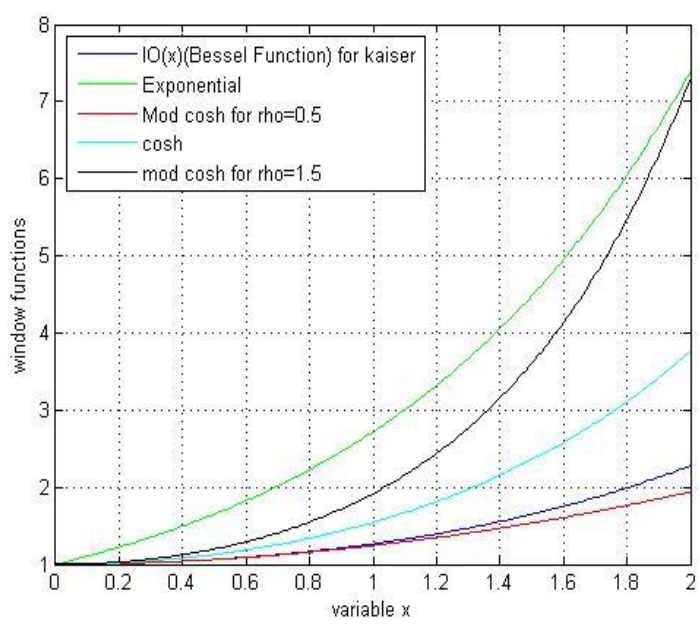

Fig 1Similar Basis Functions of Several Windows

From the above graph, it is clearly visible that all the above window functions have the same shape characteristics.

By considering the same shape characteristics of all the above window functions Hyperbolic Cosine and Exponential functions are proposed to be considered as window functions.
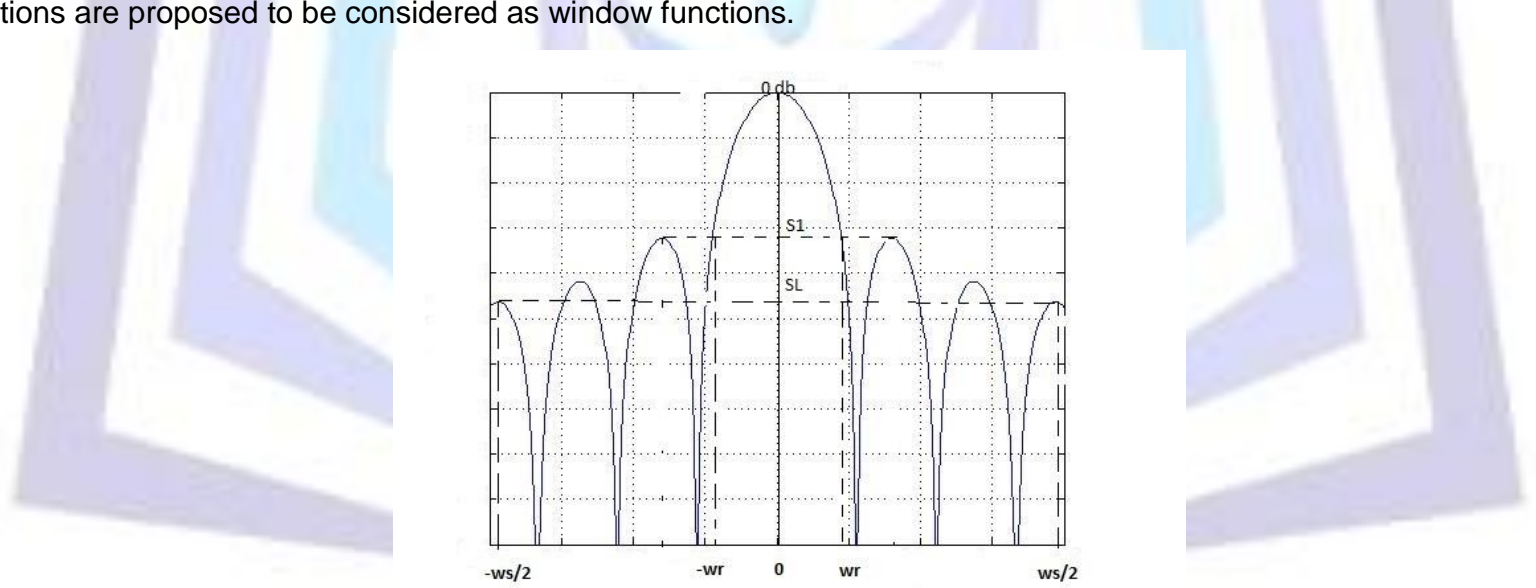

Fig. 2 General Window Function

Form the above figure[7,8],

(i) Ripple Ratio = Maximum side lobe amplitude - Main lobe amplitude $=\mathrm{S} 1 \mathrm{db}$

(ii) Side lobe Roll off Ratio = Maximum side lobe amplitude - Minimum side lobe amplitude $=\mathrm{S} 1-\mathrm{SL} d \mathrm{db}$

(iii) Main lobe width $=2 \mathrm{wr} \mathrm{rad} / \mathrm{sec}$

(iv) Normalized Main lobe width i.e. $\mathrm{D}=2 \mathrm{wr}(\mathrm{N}-1) \mathrm{rad} / \mathrm{sec}$

To provide the best filters designed by the 3-parameter Cosh window, it is important to select the optimum values for the adjustable parameters $\rho \mathrm{mc}$ and amc. The optimum choice for the adjustable parameters means that the obtained filter provides the highest minimum stop-band attenuation for the given filter length and cut-off frequency. The adjustable parameters to be optimum are selected such that two ripples (including the first one) in stop-band region are equal in amplitude. The values for optimum modified cosh window have been calculated by several iterations in MATLAB. Optimum modified cosh window has better ripple ratio[13]. 
The equations for optimum modified cosh window have been derived by varying its shaping parameters $\alpha$ and $\rho$ for the same window size empirically. The values have been find out and used to derive equations by using the Curve Fitting Tool method.

The equations are as under[13]:

$R$ ranging from $-13.26 \leq R \leq-60$

For $\mathrm{N}=51$

$$
\begin{aligned}
& \alpha_{\mathrm{mc}}=-5.532 \times 10^{-5} R^{5}-9.276 \times 10^{-3} R^{3}-0.531 R-8.446 \\
& \rho_{\mathrm{mc}}=2.526 \times 10^{-5} R^{4}+5.065 \times 10^{-3} R^{3}+0.38 R^{2}+12.46 R+160.1
\end{aligned}
$$

For $\mathrm{N}=101$

$$
\begin{gathered}
\alpha_{\mathrm{mc}}=-5.098 \times 10^{-5} R^{5}-9.869 \times 10^{-3} R^{3}-0.564 R-9.027 \\
\rho_{\mathrm{mc}}=3.197 \times 10^{-5} R^{4}+6.353 \times 10^{-3} R^{3}+0.47 R^{2}+15.53 R+193.8
\end{gathered}
$$

\section{RELATED WORK}

Pankaj Shukla, Vikas Soni and Mithilesh Kumar (2011), had analysed and compared the Modified Cosh window with other windows with respect to its spectral characteristics. They have simulated this window for FIR filters designing and find that the filters designed by proposed 3-parameter Cosh window provide higher quality in terms of the minimum stopband attenuation for a fixed order, and yield lower order for a fix minimum stopband attenuation compared to the filters designed by other windows. The proposed Hyperbolic Cosine window for 3-parameters makes more flexible as compared to the 2 parameters Hyperbolic Cosine window. It gives the better results in terms of minimum stopband attenuation, filter length, and far-end stopband attenuation with the optimum choice of the adjustable parameters otherwise the filter designed by the proposed window may perform worse results than the filter designed by 2-parameter Cosh window, although it has one more parameter. The optimum adjustable parameters which provides the highest minimum stopband attenuation are selected such that two ripples (including the first one) in stopband region are equal in amplitude. It is shown that with the optimum choices of the adjustable parameters, the

3-parameter Cosh window provides best filters compared to 2-parameter Cosh window for all transition width. The proposed window is compared with the well known windows in literature in terms of the minimum stopband attenuation, filter length or filter order and far-end stopband attenuation. The first example demonstrate that the filter designed by the proposed window produces the highest minimum stopband attenuation and the highest far-end stopband attenuation compared to the filters designed by other windows in literature for a given fix filter length and transition width. The second example shows that the required filter length, $\mathrm{N}$, of the filters designed by the proposed window is minimum among other adjustable windows in literature. It implies that the best filter provides the lowest order, N-I, is obtained by the proposed 3 parameter Hyperbolic Cosine window that is why the filter sign by the proposed window may be called as High Quality low order filter design.

K.Avci, A.Nacaroglu (2008), had proposed a new window function based on exponential function. It has been proposed that the Exponential window provides better side-lobe roll-off ratio than Kaiser window which is very useful for some applications such as beam forming, filter design, and speech processing. They had proposed exponential window function for the designing of non-recursive digital

FIR filters. Digital FIR filter designed by Kaiser window has a better far-end stopband attenuation than filter designed by the other previously well known adjustable windows such as Dolph-Chebyshev and Saramaki, which are special cases of Ultraspherical windows, but obtaining a digital filter which performs higher far-end stopband attenuation than Kaiser window will be useful. It is seen that the FIR filter designed by proposed window provides the worse minimum stopband attenuation but better far end attenuation than filter designed by well known Kaiser Window. The better far end stopband attenuation in case of Exponential window shows the figure of merit and it is used for some applications such as sub band coding and speech processing [6]. The comparison example compare this proposed window with Kaiser and previously proposed Cosh window [9] on the basis of Normalized Transition width D, Filter Length N Design Parameter $\square$, far end stopband attenuation and minimum stopband attenuation and shows that the far end stopband attenuation is maximum in FIR filters designed by Exponential window than Kaiser and Cosh windows.

K.Avci, A.Nacaroglu (2008), had proposed a modification in window based on hyperbolic cosine function with introduction of third parameter. These parameters which are varied according to the requirements of the spectral characteristics are known as shaping parameters. The parameter induced in existing 2-parameter Cosh window is 0. Now the three shaping parameters are $\mathrm{N}$, pand $\alpha$. It is observed that an increase in the new parameter results in wider mainlobe width and 
smaller ripple ratio. By choosing the suitable combinations of the two adjustable parameters for the proposed modified window, the optimum windows that yield the minimum ripple ratio for $\mathrm{N}=51$ and $\mathrm{N}=101$ are found $\mathrm{As}$ a result of the window length comparison with Kaiser and cosh windows, it is observed that the minimum window length required for satisfying a given mainlobe width and ripple ratio is obtained by the proposed modified cosh window. As an application, the proposed modified cosh window is discussed for the image processing, and compared with Kaiser, Dolph- Chebyshev, Cosh and Hamming windows in terms of the ripple ratio and contrast ratio. The simulation results show that using suitable adjustable parameters, the proposed modified cosh window can perform good results in terms of the ripple ratio and contrast ratio.

\section{CONCLUSION}

The covered work was done on studying and analyzing the proposed window performance on varying its parameters. It is found that an increase in the new parameter results in wider main-lobe width and smaller ripple ratio. The performance is counted on the basis of its spectral characteristics which are Ripple ratio, Side-lobe Roll-off ratio and Transition Width. In the result and discussion part, various plots are discussed related to modified COSH window function in time and frequency domain and its dependence on parameters. Its performance is compared with Cosh and Kaiser Windows w.r.t. spectral parameters. Specific values of proposed window parameters which are responsible for changing its shape and characteristics are derived for an OPTIMUM MODIFIED COSH WINDOW. To conclude, the results of window length comparison with Kaiser and Cosh windows, it is observed that the minimum window length required for satisfying a given main-lobe width and ripple ratio is obtained by the proposed modified Cosh window. Since the windows are suboptimal solutions, the best window is depending on the applications.

\section{REFERENCES}

[I] T. S. E1-A1i, Discrete Systems and Digital Signal Processing with MATLAB, CRC Press, 2004

[2] A. Antoniou, Digital Signal Processing: Signal, Systems, and Filters, McGraw-Hili, 2005

[3] S. W. A. Bergen and A. Antoniou, "Design of Ultraspherical window functions with prescribed spectral characteristics" EURASIP Journal on Applied Signal ProceSSing, no.I3, 2004, pp. 2053-2065

[4] F.Kaiser and R.W.Schafer, "On the use of the lo-sinh window for spectrum analysis" IEEE Trans. AcoustiCS, Speech, and Signal Processing, vol.28, no.l, 1980, pp.105-107

[5] F. Kaiser, "Nonrecursive digital filter design using IO-sinh window function" in Proc. IEEE Int. Symp. Circuits and Systems (ISCAS74), San Francisco, Calif, USA, 1974, pp.20-23

[6] S. W. A. Bergen and A. Antoniou, "Design of Nonrecursive Digital Filters Using the Ultraspherical Window Function" EURASIP Journal on Applied Signal ProceSSing, no.I2, 2005, pp. 1910- 1922

[7] A. Jain, R. Saxena, S.C. Saxena, "A simple alias-free QMF system with near-perfect reconstruction" J.indian Ins.Sci., Jan-Feb, no.12, 2005, pp. 1-10

[8] C. L. Dolph, "A Current Distribution for Broadside Arrays Which Optimizes the Relationship Between Beamwidth and Side-lobe Level" Proc.

IRE, vo1.34, pp.335-348, June 1946.

[9] T. Saramaki, "A class of window functions with nearly minimum sidelobe energy for designing FIR filters" in Proc. IEEE Int. Symp. Circuits and systems (ISCAS'89), Portland, Ore, USA, yoU, pp. 359 - 362, 1989.

[10] K. Avci, A. Nacaroglu, "Cosine hyperbolic window family with its application to FIR filter design". Proceedings of 3rd International Conference

on Information \& Communication Technologies: from Theory to Application (ICTIA'08), pp. 289-290, Damascus, Syria, April 2008.

[II] K. Avci, Arif Nacaroglu," A New Window Based on Exponential Function". Proceedings of 3rd International Conference on Information \& Communication Technologies: from Theory to Applications (ICTTA'08), pp. 289-290, Damascus, Syria, April 2008.

[12] K. Avci, N. Nakaroglu, " High Quality Low Order Nonrecursive Digital Filter Design Using Modified Kaiser Window", IEEE international Conference ,2008.

[13] Kemal Avci and Arif Nacaroglu, "Modification of Cosh window Family", ICTTA, 2008.

[14] Pankaj Shukla, Vikas Soni and Mithilesh Kumar," Non recursive digital FIR Filter design by 3-parameter Hyperbolic Cosine Window: A High Quality Low order Filter Design “, IEEE, 2011. 